Hugh Sinclair Unit of Human Nutrition, Department of Food and Nutritional Sciences, University of Reading, Reading, UK

2 MRC Epidemiology Unit, University of Cambridge School of Clinical Medicine, Cambridge, UK

3 Primary Care Diabetes and Vascular Medicine, Diabetes Research Centre, University of Leicester, Leicester, UK

Correspondence to: K S Vimaleswaran v.karani@reading.ac.uk

Cite this as: BMJ2021;372:n544 http://dx.doi.org/10.1136/bmj.n544

Published: 04 March 2021

\title{
Vitamin D and covid-19
}

\section{Benefits are possible but evidence is sparse, indirect, and inconclusive}

\author{
Karani S Vimaleswaran, ${ }^{1}$ Nita G Forouhi, ${ }^{2}$ Kamlesh Khunti ${ }^{3}$
}

The covid-19 pandemic has led to many unfounded and exaggerated claims about possible treatments. One high profile controversy has been the role of vitamin $\mathrm{D}$ in the prevention and management of covid-19, ${ }^{12}$ so the National Institute for Health and Care Excellence (NICE), Public Health England, and the Scientific Advisory Committee on Nutrition rapid guideline is timely. ${ }^{3}$

The joint guidance concludes that there is little good evidence on vitamin D and covid-19, highlights the need for further research, and supports existing government advice that adults and children in the UK should take $10 \mu \mathrm{g}$ (400 IU) a day between October and March, to optimise musculoskeletal health. It also recommends that certain populations such as minority ethnic groups, consider taking vitamin D throughout the year.

\section{What's the evidence?}

Vitamin D supplementation of 10-25 $\mu$ g a day has a modest protective effect against acute respiratory infections, ${ }^{4}$ but research on a direct effect in covid-19 is sparse. The NICE review ${ }^{5}$ included one small randomised controlled trial of vitamin $\mathrm{D}$ as treatment, ${ }^{6}$ no trials of vitamin $\mathrm{D}$ as prevention, and 12 observational studies investigating associations between serum vitamin $\mathrm{D}$ concentrations and incidence or treatment of covid-19. The one small $(n=76)$ low quality trial from Spain reported significantly reduced disease severity among patients given high dose vitamin $\mathrm{D}$ during their hospital admission. ${ }^{6}$

Two further trials not included in the NICE review reported conflicting findings. A single oral dose of $5000 \mu \mathrm{g}$ of vitamin D3 did not influence length of stay among Brazilian patients with severe covid-19 $(n=240) .{ }^{7}$ In a smaller trial from India, however, $(\mathrm{n}=40)$ patients with mild or asymptomatic covid-19 were more likely to test negative at 21 days following daily vitamin D supplementation starting at $1500 \mu \mathrm{g}$. $^{8}$ Differences in participants; type, dose, initial vitamin status, and duration of vitamin D supplementation; study endpoints; and risk of bias make interpretation of the trial evidence difficult.

Observational evidence is also inconsistent. Some, but not all, studies report an association between vitamin D deficiency and greater incidence or severity of SARS-CoV-2 infection. ${ }^{59}$ The extent of uncontrolled confounding by age, ${ }^{10}$ ethnicity, ${ }^{11}$ genetic

heterogeneity, ${ }^{12}$ and obesity ${ }^{1314}$ varies among studies, however, and probably accounts for at least some of the observed associations.

Though direct evidence of a link between vitamin D levels and covid-19 incidence or outcomes is lacking, indirect evidence of an immunomodulatory role of vitamin $\mathrm{D}$ in respiratory infections exists. Other indirect evidence includes the similarity of the risk factors for both vitamin D deficiency and severe covid-19: older age, obesity, and minority ethnicity. Also, the correlation between seasonal decline of serum concentrations of vitamin $\mathrm{D}$ and higher burden of covid-19 in high latitude countries. ${ }^{15}$ Taken together, existing evidence supports a compelling case for further research.

\section{Implications for guidance}

Although the recommended $10 \mu \mathrm{g}$ daily seems justifiable to maintain serum concentrations of 25-hydroxyvitamin D above $25 \mathrm{nmol} / \mathrm{L}$, whether this is enough to gain any immunomodulatory benefits for patients with covid-19 remains unclear. UK guidance recommending $10 \mu \mathrm{g}$ a day of vitamin D has been in existence for a while, but adherence is not guaranteed. Raising awareness of the relevance of vitamin D to musculoskeletal health is therefore appropriate, particularly during pandemic restrictions on movement. Evidence for a role in covid-19 remains suggestive only, but people may choose to take the recommended dose on the precautionary principle that it does no harm, may be beneficial, and improves bone health.

Vulnerable groups in particular need guidance on how to obtain vitamin D. Healthcare professionals can direct people at high risk to free NHS provision ${ }^{16}$ and eligible women and children to the Healthy Start scheme (https://www.healthystart.nhs.uk/).

Vegetarians and vegans need more specific guidance on sources of vitamin D supplements that match their dietary choices.

It's important that people are not falsely reassured by vitamin D supplements, and guidance must stress the importance of hand hygiene, face coverings, physical distancing, and vaccination against covid-19 in culturally and linguistically appropriate campaigns through local community groups.

Further trials evaluating vitamin D supplements in the prevention and management of covid-19 are now justified, with particular attention to different doses, baseline vitamin D levels of participants, and effects on different population subgroups and in different settings, including hospitals. Ongoing trials such as Covit $^{17}$ and Coronavit (NCTo4579640), which compares three different doses, will help inform future guidance.

Competing interests: The BM/has judged that there are no disqualifying financial ties to commercial companies. The authors declare the following other interests: NGF is an honorary consultant public health physician with Public Health England. The views expressed are her own. KK is director of the University of Leicester Centre for Black Minority Ethnic Health, trustee of the South Asian Health

Foundation, chair of the ethnicity subgroup of SAGE, and member of Independent SAGE.The BMJ policy on financial interests is here: 
https://www.bmj.com/sites/default/files/attachments/resources/2016/03/16-current-bmj-educationcoi-form.pdf.

Provenance and peer review: Not commissioned; externally peer reviewed.

1 Ali N. Role of vitamin D in preventing of COVID-19 infection, progression and severity. J Infect Public Health 2020;13:1373-80. doi: 10.1016/j.jph.2020.06.021. pmid: 32605780

2 Pereira M, Dantas Damascena A, Galvão Azevedo LM, de Almeida Oliveira T, da Mota Santana J. Vitamin D deficiency aggravates COVID-19: systematic review and meta-analysis. Crit Rev Food Sci Nutr 2020:1-9. doi: 10.1080/10408398.2020.1841090. pmid: 33146028

3 NICE. Covid-19 rapid guideline: vitamin D. https://www.nice.org.uk/guidance/ng187

4 Jolliffe DA, Camargo CA, Sluyter JD, etal. Vitamin D supplementation to prevent acute respiratory infections: systematic review and meta-analysis of aggregate data from randomised controlled trials.medRxiv 2020doi: 10.1101/2020.07.14.20152728. [Preprint.]

5 NICE. Vitamin D for covid-19: evidence reviews for the use of vitamin D supplementation as prevention and treatment of covid-19.2020. https://www.nice.org.uk/guidance/ng187/evidence/evidence-reviews-for-the-use-of-vitamin-d-supplementation-as-prevention-and-treatment-ofcovid19-pdf-8957587789.

6 Entrenas Castillo M, Entrenas Costa LM, Vaquero Barrios JM, etal. Effect of calcifediol treatment and best available therapy versus best available therapy on intensive care unit admission and mortality among patients hospitalized for COVID-19: A pilot randomized clinical study.J Steroid Biochem Mol Biol 2020;203:105751. doi: 10.1016/j.jsbmb.2020.105751. pmid: 32871238

7 Murai IH, Fernandes AL, Sales LP, etal. Effect of a single high dose of vitamin D3 on hospital length of stay in patients with moderate to severe covid-19: a randomized clinical trial. JAMA 2021. doi: 10.1001/jama.2020.26848. pmid: 33595634

8 Rastogi A, Bhansali A, Khare N, etal. Short term, high-dose vitamin D supplementation for COVID-19 disease: a randomised, placebo-controlled, study (SHADE study). Postgrad Med J 2020:postgradmedj-2020-139065. doi: 10.1136/postgradmedj-2020-139065. pmid: 33184146

9 Kaufman HW, Niles JK, Kroll MH, Bi C, Holick MF. SARS-CoV-2 positivity rates associated with circulating 25-hydroxyvitamin D levels. PLoS One 2020;15:e0239252. doi: 10.1371/journal.pone.0239252. pmid: 32941512

10 Baktash V, Hosack T, Patel N, etal. Vitamin D status and outcomes for hospitalised older patients with COVID-19. Postgrad Med J 2020:postgradmedj-2020-138712. doi: 10.1136/postgradmedj-2020-138712. pmid: 32855214

11 Khunti K, Singh AK, Pareek M, Hanif W. Is ethnicity linked to incidence or outcomes of covid-19?BM/2020;369:m1548. doi: 10.1136/bmj.m1548. pmid: 32312785

12 Anastassopoulou C, Gkizarioti Z, Patrinos GP, Tsakris A. Human genetic factors associated with susceptibility to SARS-CoV-2 infection and COVID-19 disease severity. Hum Genomics 2020;14:40. doi: 10.1186/s40246-020-00290-4. pmid: 33092637

13 Syed AA, Soran H, Adam S. Obesity and covid-19: the unseen risks. BMJ2020;370:m2823. doi: 10.1136/bmj.m2823. pmid: 32675216

14 Vimaleswaran KS, Berry DJ, Lu C, etalGenetic Investigation of Anthropometric Traits-GIANT Consortium. Causal relationship between obesity and vitamin D status: bi-directional Mendelian randomization analysis of multiple cohorts. PLoS Med 2013;10:e1001383. doi: 10.1371/journal.pmed.1001383. pmid: 23393431

15 Kohlmeier M. Avoidance of vitamin D deficiency to slow the COVID-19 pandemic. BMJ Nutr Prev Health 2020;3:67-73. doi: 10.1136/bmjnph-2020-000096. pmid: 33230496

16 NHS. Get vitamin D supplements 2020. https://www.nhs.uk/conditions/coronavirus-covid19/people-at-higher-risk/get-vitamin-d-supplements/.

17 Annweiler C, Beaudenon M, Gautier J, etalCOVIT-TRIAL study group. COvid-19 and high-dose VITamin D supplementation TRIAL in high-risk older patients (COVIT-TRIAL): study protocol for a randomized controlled trial. Trials 2020;21:1031.

doi: 10.1186/s13063-020-04928-5. pmid: 33371905 